\title{
Serological investigations in retrospective diagnosis of malaria
}

\author{
C C DRAPER, S S SIRR
}

\section{Summary and conclusions}

Sera were obtained in 415 known cases of malaria (88 residents, 327 immigrants) at different times after diagnosis. Three antigens were used in the indirect fluorescence antibody test to detect antibodies to either Plasmodium falciparum or $P$ vivax. Results in residents and immigrants were analysed separately. Most residents had detectable antibodies within one week after an attack, which began to wane after a month. The strongest reactions were obtained in cases of falciparum malaria with the homologous antigen and in cases of vivax malaria with $P$ fieldi. The overall pattern of results was the same in the immigrants but the proportions positive for malaria antibodies, mean titres, persistence of antibodies, and the cross-reactions were usually greater.

Testing for malaria antibodies is probably of value in the retrospective differential diagnosis of malaria in patients who have not been exposed to malaria before but must be interpreted with caution in others.

\section{Introduction}

Recently there has been a steady increase in malaria in the travelling public but this has been greatest in immigrants. Over 2000 cases were reported in Britain in 1979, but the total was almost certainly greater because of incomplete notification and missed diagnoses (Malaria Reference Laboratory, London School of Hygiene and Tropical Medicine, personal communication). A blood film should be taken from anyone whose symptoms and history of travel suggest malaria; when these suggest the possibility of a Plasmodium falciparum infection in a severely ill patient treatment should not be delayed while awaiting the results of examination.

Ross Institute, London School of Hygiene and Tropical Medicine, London WC1E 7HT

C C DRAPER, DM, senior lecturer

S S SIRR, junior technical officer
In clinical practice serology has no place in diagnosing acute malaria but in certain circumstances can be useful for retrospective diagnosis. Patients who have been in the tropics often present with a history of malaria symptoms but without parasites having been found on microscopy. This may be because a blood film was never made or because they had taken antimalarial drugs at a dosage sufficient to depress parasitaemia to undetectable levels before this was done. In such cases serological investigations may confirm or exclude malaria and, as well as satisfying curiosity, suggest by exclusion that other causes of the fever should be sought. It is important to confirm an infection with $P$ vivax, since this would indicate that a course of primaquine should be given to eradicate the persistent exoerythrocytic stage, which can cause relapses.

$\mathrm{Up}$ to 500 serum samples a year are received at our laboratory with a request for serological investigations for malaria, so there is clearly a demand for this service. We have attempted to define the scope and limitations of such investigations in a sample of the type of cases that are seen in Britain.

\section{Patients, methods, and results}

With the collaboration of many doctors over four years sera were obtained from known cases of malaria, proved by finding parasites in the blood, at different times after diagnosis and during treatment. Sera were examined at fourfold dilutions, from $1 / 16$ to $1 / 16384$, with the indirect fluorescence antibody test, which remains the most specific and sensitive method for detecting malaria antibodies. ${ }^{1}$ The following antigens were used: $P$ falciparum obtained from infected chimpanzees or aotus monkeys or, more recently, from continuous culture; $P$ brasilianum, which is closely related to $P$ malariae, from chimpanzees; and $P$ fieldi from Macaca mulatta monkeys. We have found that $P$ fieldi, which was reportedly antigenically related to human malaria parasites, ${ }^{2}$ is particularly closely related to $P$ vivax.

The table gives the results. Those from travelling residents were analysed separately from those of immigrants because the histories of malaria were probably different. All of the residents, so far as we know, were suffering from first attacks of malaria. The data were mostly cross-sectional and contained only a few serial observations. Most of the residents had detectable antibodies within one week after an attack of malaria, which generally began to wane after a month as shown by a fall in the mean titres. An outstanding feature,

Results of indirect fluorescence antibody tests with different antigens in known cases of malaria in residents and immigrants

Infecting parasite and time since clinical onset of symptoms

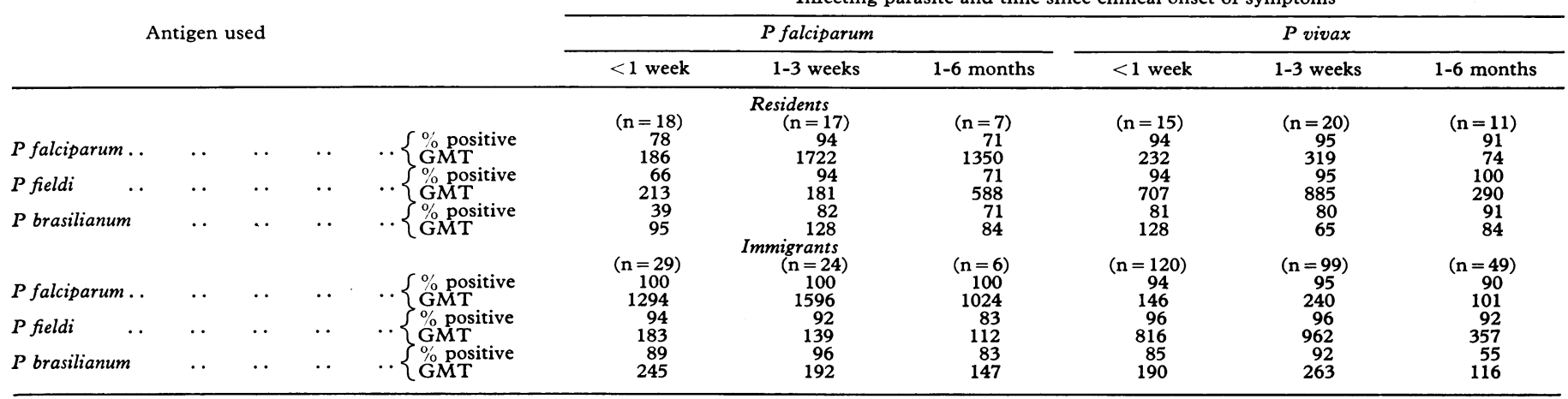

GMT $=$ Geometric mean reciprocal titre. 
however, was the wide range of titres in different people at the same time after infection (from $1 / 16$ to $1 / 4096$ ). The strongest reactions were obtained in cases of falciparum malaria with the homologous antigen, and in cases of vivax malaria with $P$ fieldi. Sera from patients with $P$ falciparum showed considerable cross-reaction to $P$ vivax and vice versa; sera from both groups showed less cross-reaction to $P$ brasilianum.

In the immigrants, who included subjects with a wide range of malaria histories, the overall pattern was the same as in the residents but the proportions positive for malaria antibodies, the mean titres, the persistence of antibodies, and the cross-reactions were usually greater.

Eight cases of $P$ malariae infection in residents and 14 such cases in immigrants were also studied. All patients had detectable antibodies in the first week after a positive blood film, which persisted for up to six months after treatment with reactions against $P$ fieldi as strong as against $P$ brasilianum.

\section{Discussion}

The amount of antibody detected after an attack of malaria depends on the amount of antigenic stimulus which is related to the species of parasite, how soon and how much treatment is given, the occurrence of relapses, the history of malaria, and immunological capability and memory.

In non-immune residents, most of whom will probably have received some treatment soon after the onset of symptoms, the indirect fluorescence antibody test will detect antibodies in most during the first week after onset, but the proportion positive for malaria antibodies will increase from one to four weeks after onset and then decrease. If several antigens are used it is usually possible to identify the species of parasite by the highest titre, although there are considerable crossreactions between all the human and some simian plasmodia. Because of the variability of the response it is difficult to be precise about the time of the attack from the antibody titre alone. Higher titres (1/256 and over) probably indicate an attack within the past six months, but lower titres can be said to indicate only that an attack has occurred. Similar conclusions were reached in a study of treated cases of malaria in US servicemen. ${ }^{3}$

The higher figures in immigrants may be related to delay in seeking treatment but in most cases were probably due to an anamnestic response after previous malaria. This and the persistence of antibodies from past infections make interpretation of serological results alone in such people difficult, and an assessment of each case must take into account symptoms, antibody titre, and recent and past residence and travel. For example, a visitor from a hyperendemic malaria area in Africa may have high titres of antibodies reactive to all antigens, which may be associated with a low-grade asymptomatic infection that is undetectable microscopically. On the other hand an immigrant from Asia who has caught malaria on a return visit to his home will have an antibody response more like that of a resident, but will probably have higher titres and more cross-reactions because of having already had malaria.

After repeated attacks, malaria antibodies may be detectable for many years. ${ }^{1}$ This was shown by a study of sera from patients with neurosyphilis who had been treated with prolonged attacks of malaria, sometimes with several species, before undergoing radical treatment. Fourteen out of 18 patients $(78 \%)$ still had detectable antibodies $11-20$ years after their last episode of malaria, and 12 out of $17(70 \%)$ after 20 years or more, including one after 30 years. These results extend the maximum period recorded for the persistence of malaria antibodies. ${ }^{4}$

We conclude that the determination of malaria antibodies is of value in the retrospective differential diagnosis of fevers in patients who have not been exposed to malaria before but must be interpreted with care in others.

Neurosyphilitic sera was kindly provided for study by Professor L J Bruce-Chwatt, Miss M Maryon, and the late Mr P G Shute.

\section{References}

${ }^{1}$ Bruce-Chwatt LJ, Dodge JS, Draper CC, Topley E, Voller A. Seroepidemiological studies on population groups previously exposed to malaria. Lancet $1972 ; \mathrm{i}: 512-5$

${ }^{2}$ Collins WE, Jeffery GM, Guinn E, Skinner JC. Fluorescent antibody studies in human malaria IV cross reactions between human and simian malaria. Am f Trop Med Hyg 1966;15:11-5.

3 Wilson M, Sulzer AJ, Runcik K. Malaria antibody patterns as determined by the IFA test in US servicemen after chemotherapy. Am $\mathcal{F}$ Trop Med Hyg 1970;19:401-4.

${ }^{4}$ Collins WE, Skinner JC, Jeffery GM. Studies on the persistence of malarial antibody response. Am $\mathcal{F}$ Epidemiol 1968;87:592-8.

(Accepted 24 March 1980)
ONE HUNDRED YEARS AGO We are glad to hear that, for sanitary reasons adduced in the Report of the United Synagogue of British Jews, the Council have resolved that the use of mourners' cloaks at funerals be discontinued. It is to be hoped that the palls will shortly also be abolished. We earnestly recommend the general community to follow an example dictated by sound common sense and justified by the teachings of sanitary science.

"Public attention has lately been called to some of the probable means by which contagion is diffused. The Committee are of opinion that the palls and mourners' cloaks afford such a means for spreading infection. These appurtenances are of course used indiscriminately in cases of death from infectious and non-infectious disease, and almost necessarily carry with them from house to house the insidious germs of the most fatal maladies. The mischief which may be propagated by contact with'these paraphernalia is incalculable. The Committee recommend the Council to resolve that they be discontinued at or in connection with the funerals of the United Synagogue. The simplicity with which funerals should be conducted has been generally recognised both within and without the community. The solemnity of a funeral can receive no augmentation from the use of a pall, nor can the feelings of the mourners be affected in any degree by the employment of a black cloak. The first was introduced to hide from public view the white deal of which coffins for Jewish use are, in the majority of instances, made; and the latter originated in the desire to cover the garments of poorer mourners, who might feel ashamed to attend the funerals of their relatives, or the synagogue on the Sabbath during the week of mourning, in their usual attire. The Committee believe that, with the spread of intelligence, these reasons have ceased to be operative. Educated public opinion sees nothing disrespectful, but, on the contrary, something commendable, in the absence of decorative appliances which distinguishes a Jewish funeral; and the sympathy and condolence extended to all who mourn must render the use of a black cloak to conceal the mourner's attire superfluous. There may be some in the congregation who will object to relinguishing a custom which time has consecrated to them; but the Committee trust that the Council will take this opportunity of teaching and leading the minds of the congregants in such grave circumstances and considerations as are involved in the possible introduction and spread of contagious diseases in their households." (British Medical fournal, 1880.)

\section{Correction}

\section{High-pressure water jet injury}

We regret that an error occurred in this article by $\mathrm{Mr} \mathrm{J} \mathrm{L} \mathrm{M} \mathrm{de} \mathrm{Beaux}$ (14 June, p 1417). A jet velocity of 900 miles an hour, referred to in the third line, was wrongly converted to $559 \mathrm{~km}$ : it should have read $1448 \mathrm{~km}$. 